\title{
Protocol based treatment for hypertension in rural/urban health training centres
}

\author{
Savitha AK \\ Dept. of Community Medicine, Sree Balaji Medical College \& Hospital, Chennai, Tamil Nadu
}

Email: drsavitha22@gmail.com

\section{Introduction}

The rural and urban health training centres provide curative and follow up care of diagnosed hypertension case. They also provide defined interventions for prevention and screening of the disease within their catchment areas. Apart from the availability of essential medicine and simple medical equipment, treatment protocols are essential for appropriate management for non communicable diseases like hypertension. The benefits of adoption and use of standardized, evidencebased protocol for management of hypertension are

1. Protocols reduce clinical variability that is outside the bounds of evidence-based practice.

2. A protocol can better enlist and enable all members of the health care team to reinforce the importance of blood pressure control and the value of adherence to healthy habits, medications, and selfmonitoring, allows qualified staff to advance patients safely and efficiently along the treatment pathway, ensures that the supervising clinician is consulted if clinical exceptions occur.

3. Algorithms can be incorporated into electronic health records through clinical decision support tools to facilitate quality improvement.

4. A protocol can result in a more efficient and cost effective selection of medications and treatment approaches.

5. Standardized treatment facilitates evaluation, both the quality and impact quality of care.

6. Adopting a standardized treatment approach sends a strong signal to clinical staff that hypertension control is a priority.
The health centres should try to deliver a defined package of services to prevent and control hypertension consisting of: information, education and communication related to healthy lifestyle and proper nutrition and regular medical check-ups and management.

It is important to adopt standardized treatment protocols for improving blood pressure control. Simple, evidence-based treatment protocols can have a powerful impact in improving control and treatment options, in timely follow-up with patients, with effective reporting system. A variety of evidence-based hypertension treatment protocols are available for practices and health care systems to select from, or a custom protocol can be developed depending upon the resources settings.

The recommended elements of a treatment protocol for hypertension are,

1. Clarity and simplicity

2. Lifestyle modification

3. Treatment by stage of hypertension

4. Time interval to titration and reassessment

5. Use of low-cost 1st-line treatment

6. Exclusions and suggestions for medications based on concurrent medical conditions

7. Recommended lab tests

8. Reminder of the underlying causes of non-essential or secondary hypertension

9. Adherence enhancing approaches such as fixed dose and/or combination drugs

10. Indications for referral to higher centres

11. Supporting references

12. Congruent with current guidelines (e.g. JNC - 8)

\section{Proposed Solution}

A sample of evidence based hypertension screening and management protocol for adults that can be used in the urban / rural health training centre is given below.

\section{Protocol for screening hypertension}

\begin{tabular}{|c|c|c|}
\hline \multirow{2}{*}{$\begin{array}{l}\text { SBP >- } 140 \mathrm{mmHg} \text { or DBP >- } \\
\text { 90mmHg Reconfirm with } \\
\text { second reading }\end{array}$} & & \\
\hline & $\begin{array}{l}\text { SBP } 120-130 \mathrm{mmHg} \text { or } \\
\text { DBP } 80-89 \mathrm{mmHg}\end{array}$ & $\begin{array}{l}\text { SBP }<120 \mathrm{~mm} \mathrm{Hg} \text { and DBP } \\
<80 \mathrm{~mm} \mathrm{Hg}\end{array}$ \\
\hline $\begin{array}{l}\text { Diagnosed as } \\
\text { hypertension }\end{array}$ & $\begin{array}{l}\text { Diagnosed as pre } \\
\text { hypertension }\end{array}$ & $\begin{array}{l}\text { Diagnosed as } \\
\text { nonhypertensive }\end{array}$ \\
\hline
\end{tabular}




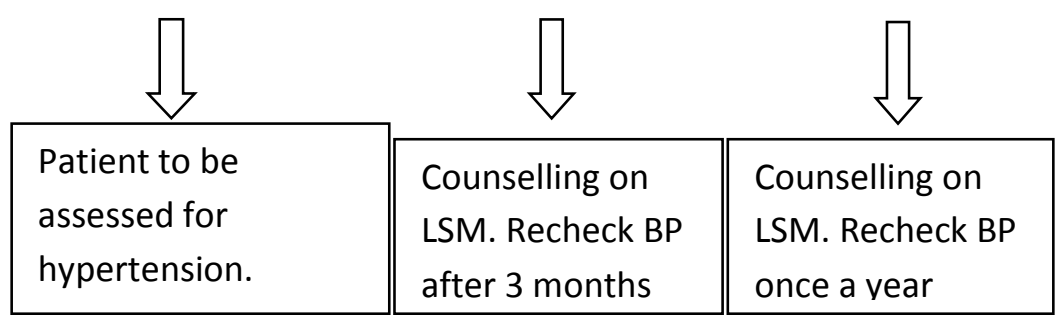

\section{Protocol for treatment of hypertension}

- A detailed history for chest pain/ angina/ischaemic heart disease/stroke/transient ischaemic heart disease/congestive cardiac failure/diabetes.

- To do urine albumin, serum creatinine, serum cholesterol, blood sugar and ECG.

Goal blood pressure : $<140 / 90 \mathrm{mmhg}$ for patients with hypertension

$<130 / 80 \mathrm{mmhg}$ for patients with diabetes or chronic kidney disease

Patients with hypertension already on treatment

- If systolic BP <140mmhg \& diastolic BP $<90 \mathrm{mmhg} \rightarrow$ Advise to continue the same treatment and counselling on LSM $\rightarrow$ Regular follow up

- If systolic BP >- 140mmhg and/or diastolic BP >$90 \mathrm{mmhg} \rightarrow$ follow the guidelines below of newly detected hypertensive patients to achieve goal blood pressure.

Newly detected patients with hypertension

Stages of Hypertension: The patients with systolic Blood Pressure 140 - $159 \mathrm{~mm} \mathrm{Hg}$ and / or Diastolic Blood Pressure 90-99 $\mathrm{mm} \mathrm{Hg}$ are classified as "stage I Hypertension". The patients with systolic BP > $160 \mathrm{~mm}$ $\mathrm{Hg}$ and / or Diastolic BP $>100 \mathrm{~mm} \mathrm{Hg}$ are classified as "stage II Hypertension".

Stage I Hypertension with risk factors:

1. If patients fall under stage I Hypertension category, then assess for risk factors like age $>55$ years, male, smoking, Family History of premature CVD, BMI $>25 \mathrm{~kg} / \mathrm{m} 2$, diabetes. If any one or more risk factors present, then start a sub-optimal dose of tablet Amlodipine $2.5 \mathrm{mg}$ per day along with counseling on Life Style Modifiation (LSM) and advise to recheck BP after two weeks.

2. If his / her BP is $<140 / 90 \mathrm{~mm} / \mathrm{Hg}$ after two weeks of LSM \& T. Amlodipine $2.5 \mathrm{mg} /$ day, advise patient to continue on Life Style Modifiation and continue same dose of T. Amlodipine.

Advise patients to come for follow-up. If the BP is $\geq 140 / 90 \mathrm{~mm} / \mathrm{Hg}$ after two weeks of LSM \& T. Amlodipine $2.5 \mathrm{mg} /$ day, then increase the dose of $\mathrm{T}$. Amlodipine up to $10 \mathrm{mg} /$ per day in two divided doses. Recheck BP after two weeks. If goal BP is not achieved, then consider to add second drug.
Stage I Hypertension without risk factors:

1. If there is no risk factor, provide only counseling on LSM and advise the patient to recheck his / BP after 2 weeks.

2. If his/ her BP is normal after two weeks of LSM, advise patient to continue on Life Style Modifiation and to come for annual followup or to report if any symptoms appear.

3. If $\mathrm{BP}$ is $\geq 140 / 90 \mathrm{~mm} / \mathrm{Hg}$ after two weeks of LSM, advise patient to continue on Life Style Modifiation and start on minimal dose of $\mathrm{T}$. Amlodipine $2.5 \mathrm{mg}$ per day. Advise patients to come for followup

Stage II Hypertension:

1. For all patients with stage II Hypertension (Systolic BP of $\geq 160 \mathrm{~mm} \mathrm{Hg}$ and / or Diastolic BP of $\geq 100$ $\mathrm{mm} / \mathrm{Hg}$ ) start $\mathrm{T}$. Amlodipine $5 \mathrm{mg}$ per day and advise counselling on LSM. Instruct the patient to recheck BP after 2 weeks.

2. If BP is $<140 / 90 \mathrm{~mm} / \mathrm{Hg}$ after 2 weeks of LSM and T. Amlodipine 5mg, advise to continue the same treatment.

3. If $\mathrm{BP} \geq 140 / 90 \mathrm{~mm} \mathrm{Hg}$ increase the dose of $\mathrm{T}$. Amlodipine to maximum of $10 \mathrm{mg}$ per day with counseling on LSM and advise to recheck BP after 2 weeks.

4. After 2 weeks if goal blood pressure is achieved, continue the same treatment. Is goal BP is not achieved then add second drug as mentioned in the protocol for treatment of Hypertension.

Criteria for starting second drug

- If Goal BP is not achieved with maximum dose of T. Amlodipine 10mg per day with LSM; then add a "second drug".

- Before adding a second drug assess whether the patient has any co existing morbidity along with Hypertension such as Diabetes, Ischaemic Heart Disease (IHD), Chronic Kidney Disease, Bronchial asthma, Chronic obstructive Pulmonary Diseases (COPD) etc.,

- Add appropriate second drug according to the patient's co-morbidity.

Treatment guidelines to be followed for different category of patients viz., Isolated Hypertension not controlled with maximum dose of T. Amlodipine, HT with DM, HT with IHD, HT with CKD and HT with COPD (Bronchial asthma).

a. If patient has Isolated Hypertension not controlled with T. Amlodipine 10mg per day: Continue with 
maximum dose of T. Amlodipine 10mg per day and counsel on LSM + Add a Second Drug - either Diuretic, T. Hydrohlorothiazide $25 \mathrm{mg}$ once daily (or) add ACE Inhibitor T.Enalapril 2.5-20 mg per day (check Sr. Creatinine value is $<2 \mathrm{mg} / \mathrm{dl}$ ) (or) add Beta Blocker, T. Atenolol 25-100 mg per day in divided doses.

b. If patient has Hypertension with Diabetes Mellitus: To add T. Enalapril 2.5-20 $\mathrm{mg}$ per day (if Sr.Creatinine is $<2 \mathrm{mg} / \mathrm{dl}$ ).

c. If patient has Hypertension with IHD: To add T. Atenolol 25-100 mg per day, with T. Aspirin $75 \mathrm{mg}$ od and T. Isosorbitrate dinirate 5$10 \mathrm{mg}$ tds.

d. If patient has Hypertension with Chronic kidney Diseases: Avoid Enalapril if serum Creatinine $>2 \mathrm{mg}$. Add T. Atenolol 25-100 mg per day (But cautiously use it in Peripheral Vascular Disease).

e. If patient has Hypertension with Bronchial asthma or COPD: Avoid Atenolol in COPD. Add T. Enalapril 2.5 -20 mg per day (check Sr. Creatinine value is $<2 \mathrm{mg} / \mathrm{dl}$ ) (or) T. Hydrochlorothiazide 25 mg od.

\section{Counseling on Life Style Modifiation (LSM) \\ should focus on key facts mentioned below:}

1. Advice to stop smoking and use of tobacco related products.

2. Increasing physical activity / exercise.

3. Maintaining ideal body weight.

4. Adopt a healthy dietary habit.

5. Advice to avoid stress.

6. Avoid overeating and overweight.

7. Maintaining normal blood pressure and normal blood sugar.

8. Advice to avoid alcohol consumption

Note: In case of suspected target organ damage or complications the patient should be referred to higher facility for further management.

(Reference: Clinical manual on NCD interventions for medical and para medical staff, 2012.,Program for prevention, screening and treatment of NCDs., TNHSP, Department of Health and Family Welfare, Government of Tamil Nadu),

\section{Recommendations}

- To develop such protocols which will be a key tool in safe and swift control of blood pressure for hypertensive patients

- Training program for medical and paramedical staff for effective use of protocol to ensure control of blood pressure

- To fully use the expertise and scope of practice of every member of the health care team: physician, nurse, pharmacist, field workers and others

- Include patient as an essential member of team.

\section{Conclusion}

Simply adopting a protocol does not mean that it will be used. Effective implementation requires continuous analysis and feedback on critical indicators such as the proportion of adults who are documented to have hypertension and the proportion of control achieved by each clinician or care team. Standardized treatment will help clinicians, teams, and patients achieve and maintain healthy blood pressures and thereby prevent complications like myocardial infarction and stroke. 